\title{
A HYPERBOLIC, EXTENDED JACOBIAN INVERSE KINEMATICS ALGORITHM FOR MOBILE MANIPULATORS
}

\author{
Krzysztof Tchoń and Janusz Jakubiak
}

\author{
Institute of Engineering Cybernetics \\ Wroctaw University of Technology \\ ul. Janiszewskiego 11/17, \\ 50-372 Wrockaw, Poland \\ email: tchon |jjakubia@ict.pwr.wroc.pl
}

\begin{abstract}
A contribution of this paper consists in deriving a repeatable, extended Jacobian inverse kinematics algorithm for mobile manipulators. The endogenous configuration space approach has been assumed as a guideline. An endogenous configuration of a mobile manipulator includes controls of the platform and joint positions of the onboard manipulator. After a suitable truncation of Fourier series representing platform controls a band-limited version of this algorithm is obtained. By reference to the shape of the invariant manifold in the band-limited situation the algorithm is called hyperbolic. Performance of this algorithm is illustrated with computer simulations involving a kinematic car-type mobile platform endowed with an RTR manipulator. Copyright (C)2005 IFAC
\end{abstract}

Keywords: Mobile robots, robot kinematics, inverse kinematic problem, robot control

\section{INTRODUCTION}

In this paper by a mobile manipulator we shall understand a robotic system composed of a nonholonomic mobile platform and a holonomic manipulator fixed to the platform. The field of mobile manipulators presents a challenge from the viewpoint of modeling, motion planning and control algorithms. On the other hand, due to their enhanced mobility and manipulability properties mobile manipulators are indispensable tools for space and service robotics. There exists a rich literature devoted to mobile manipulators, pioneered by an application in space robotics (Papadopoulos and Dubowsky, 1991). A thorough review of this literature up to 2002 has been made in (Tchoń and Jakubiak, 2003), and for the sake of space it will not be repeated here. This being so, we restrict only to mentioning papers that have appeared recently. In (Bayle et al., 2003) manipulability measures for mobile manipulators are defined and applied to the motion planning problem. The paper (Duleba and Sasiadek, 2003) proposes a solution to the motion planning problem for mobile manipulators by means of the Newton algorithm with control energy optimization. In (Galicki, 2003) an optimization theory based inverse kinematics algorithm for mobile manipulators has been set forth, capable of avoiding singularities and obstacles in the taskspace. The publication (Mazur, 2004) proposes an adaptive taskspace path following algorithm accounting for the kinematics and the dynamics of a mobile manipulator.

In (Tchoń and Muszyński, 2000; Tchoń and Jakubiak, 2003) we have developed a new approach to mobile manipulators, referred to as the endogenous configuration space approach. A fundamental concept underlying this approach is the endogenous configuration that consists of control functions of the platform and joint positions of the aboard manipulator. In particular, the endogenous configuration space approach provides a natural definition of repeatability for mobile manipulators (Tchon, 2002). In order to explain 
this concept in some details, let us consider a Jacobian inverse kinematics algorithm for mobile manipulators (Tchoń et al., 2003; Tchoń and Jakubiak, 2003). Such an algorithm has the form of a taskspace error driven dynamic system, operating on the endogenous configuration space in such a way that to every desirable point in the taskspace the algorithm assigns an endogenous configuration in which the mobile manipulator reaches this point. When processing a sequence of inverse kinematic problems the algorithm uses the solution of a given problem as an initial condition for the next problem. Suppose that a certain problem repeats in the sequence several times. The algorithm that each time returns the same solution of this problem, irrespective of the initial conditions, is referred to as repeatable. Repeatability may be described concisely as the property that a closed path in the taskspace generates a closed path in the endogenous configuration space. A geometric interpretation of repeatability is the following: outside singularities the kinematics endow the endogenous configuration space with the structure of a fiber bundle over the taskspace, such that the inverse kinematic algorithm defines a connection on this bundle. If the connection has trivial holonomy group, the algorithm is repeatable. It has been shown that an inverse kinematics algorithm for mobile manipulators is repeatable if its associated distribution is involutive, so integrable (Tchoń, 2002). This means that after choosing an initial condition, the algorithm operates on a fixed integral manifold of the associated distribution. This being so, in order to design a repeatable inverse kinematics algorithm for mobile manipulators one needs to find the associated distribution annihilated by an augmenting kinematics map such that level sets of this map coincide with integral manifolds of the distribution. Notice that exactly the same method has been applied to stationary manipulators resulting in the extended Jacobian inverse kinematics algorithm that has the property of repeatability (Baillieul, 1985; Roberts and Maciejewski, 1992).

In this paper, using a characterization of repeatability provided in (Tchoń, 2002), we define and examine a specific repeatable, extended Jacobian inverse kinematics algorithm for mobile manipulators. With reference to its invariant manifolds this algorithm is called hyperbolic. Having approximated platform control functions by means of truncated Fourier series we obtain a band-limited version of the hyperbolic algorithm with invariant manifolds determined by a hyperbolic relationship between the Fourier coefficients of platform controls and joint positions of the aboard manipulator. Performance of the algorithm has been illustrated with computer simulations involving a kinematic car-type platform equipped with an RTR manipulator. We have noticed that, although in the process of disretization repeatability of the hyperbolic algorithm may be lost, the algorithm remains convergent in a satisfactory way.
The composition of this paper is the following. In section 2 we briefly summarize the concept of Jacobian extension, and introduce the hyperbolic extended Jacobian inverse kinematics algorithm for mobile manipulators. Band-limited version of this algorithm is given in section 3. Section 4 presents results of computer simulations. The paper is concluded with section 5.

\section{BASIC CONCEPTS}

We shall study mobile manipulators consisting of a nonholonomic mobile platform and a holonomic onboard manipulator. On assumption that the platform motion obeys some velocity constraints (e.g. not permitting longitudinal or lateral slip of platform's wheels), the kinematics of such a mobile manipulator can be represented by means of a driftless control system with outputs,

$$
\left\{\begin{array}{l}
\dot{q}=G(q) u=\sum_{i=1}^{m} g_{i}(q) u_{i} \\
y=k(q, x)
\end{array}\right.
$$

where $q=\left(q_{1}, \ldots, q_{n}\right) \in R^{n}$ is a vector of generalized coordinates of the platform, $x=\left(x_{1}, \ldots, x_{p}\right) \in R^{p}$ denotes the joint positions of the aboard manipulator, and $y=\left(y_{1}, \ldots, y_{r}\right) \in R^{r}$ defines taskspace coordinates of the end effector. In most cases $m \leq n$ and $m \leq r$. In accordance with the endogenous configuration space approach we introduce the endogenous configuration space $X=L_{m}^{2}[0, T] \times R^{p}$ of the mobile manipulator comprising admissible controls $u(\cdot)$ of the platform and joint positions $x$ of the manipulator. $X$ is a Hilbert space with inner product

$$
<\left(u_{1}(\cdot), x_{1}\right),\left(u_{2}(\cdot), x_{2}\right)>=\int_{0}^{T} u_{1}^{T}(t) u_{2}(t) d t+x_{1}^{T} x_{2} .
$$

To every endogenous configuration $(u(\cdot), x) \in X$ there correspond two trajectories: $q(t)=\varphi_{q_{0}, t}(u(\cdot))$ - a trajectory of the platform, and an end effector trajectory $y(t)=k(q(t), x)$. We shall assume the existence of $q(t)$ for every $t \in[0, T]$. Departing from the representation (1) we define the instantaneous kinematics

$$
K_{q_{0}, T}: X \rightarrow R^{r}
$$

of the mobile manipulator as the input-output map of system (1)

$$
K_{q_{0}, T}(u(\cdot), x)=y(T)=k\left(\varphi_{q_{0}, T}(u(\cdot)), x\right) .
$$

The instantaneous kinematics determine reachable at $T$ end effector positions and orientations of the mobile manipulator subject to the control $(u(\cdot), x)$, provided that the platform starts from $q_{0}$.

To proceed further we need to define the analytic Jacobian

$$
\begin{gathered}
J_{q_{0}, T}(u(\cdot), x)(v(\cdot), w)=D K_{q_{0}, T}(u(\cdot), x)(v(\cdot), w)= \\
C(T, x) \int_{0}^{T} \Phi(T, s) B(s) v(s) \mathrm{d} s+D(T, x) w
\end{gathered}
$$


of the mobile manipulator as a derivative of the kinematics at the configuration $(u(\cdot), x)$. The Jacobian may be identified with the input-output map of the variational system

$$
\dot{\xi}=A(t) \xi+B(t) v, \eta=C(t, x) \xi+D(t, x) w,
$$

defined as the linear approximation (1) along a triple $(u(t), x, q(t))$, initialized at $\xi_{0}=0$, with structure matrices

$$
\begin{aligned}
& A(t)=\frac{\partial}{\partial q}(G(q(t)) u(t)), B(t)=G(q(t)), \\
& C(t, x)=\frac{\partial k}{\partial q}(q(t), x), \quad D(t, x)=\frac{\partial k}{\partial x}(q(t), x),
\end{aligned}
$$

and transition matrix $\Phi(t, s)$ satisfying the evolution equation $\frac{\partial}{\partial t} \Phi(t, s)=A(t) \Phi(t, s), \quad \Phi(s, s)=I_{n}$.

In what follows we shall be confined to the case of $p=r$. A repeatable inverse kinematics algorithm should operate on an invariant submanifold of the endogenous configuration space, and simultaneously provide a right inverse of the analytic Jacobian. The invariant manifolds will be introduced as level sets of an augmenting kinematics map

$$
H_{q_{0}, T}: X \longrightarrow L_{m}^{2}[0, T] .
$$

A pair composed of the kinematics (2) and of the map (5) is referred to as the extended kinematics

$$
\left(H_{q_{0}, T}, K_{q_{0}, T}\right): X \rightarrow X
$$

of the mobile manipulator. We shall examine the following simple augmenting map

$$
H_{q_{0}, T}(u(\cdot), x)(t)=\left(x_{1} u_{1}(t), x_{2} u_{2}(t), \ldots, x_{m} u_{m}(t)\right) .
$$

It follows that on the level sets of this map the ith component of the platform control is proportional to a fixed function from $L^{2}[0, T]$, with proportionality coefficient equal to $x_{i}$. Having substituted the map (7) into the extended kinematics (6), we get the following extended Jacobian

$$
\begin{gathered}
\bar{J}_{q_{0}, T}(u(\cdot), x)(v(\cdot), w)=\left(D H_{q_{0}, T}(u(\cdot), x)(v(\cdot), w),\right. \\
\left.C(T, x) \int_{0}^{T} \Phi(T, s) B(s) v(s) d s+D(T, x) w\right),
\end{gathered}
$$

where

$$
\begin{aligned}
& D H_{q_{0}, T}(u(\cdot), x)(v(\cdot), w)(t)= \\
& \quad\left(w_{1} u_{1}(t)+x_{1} v_{1}(t), \ldots, w_{m} u_{m}(t)+x_{m} v_{m}(t)\right) .
\end{aligned}
$$

In a region of the endogenous configuration space where the extended Jacobian is invertible we propose the following definition

$$
J_{q_{0}, T}^{E \#}(u(\cdot), x) \eta=\bar{J}_{q_{0}, T}^{-1}(u(\cdot), x)(0(\cdot), \eta),
$$

of a right inverse of the analytic Jacobian, where $\eta \in$ $R^{r}$. Now letting

$$
\left(J_{q_{0}, T}^{E \#}(u(\cdot), x) \eta\right)(t)=\left(\begin{array}{c}
v(t) \\
w
\end{array}\right)
$$

we deduce that

$$
\bar{J}_{q_{0}, T}(u(\cdot), x)\left(\begin{array}{c}
v(t) \\
w
\end{array}\right)=\left(\begin{array}{l}
0 \\
\eta
\end{array}\right) .
$$

By (8), (9) this is equivalent to

$$
w_{i} u_{i}(t)+x_{i} v_{i}(t)=0, \text { for } i=1, \ldots, m,
$$

and

$$
C(T, x) \int_{0}^{T} \Phi(T, s) B(s) v(s) d s+D(T, x) w=\eta .
$$

This being so, from (11) we compute

$$
v(t)=-\operatorname{diag}\left\{\frac{u_{i}(t)}{x_{i}}\right\}_{m} \bar{w}_{m},
$$

where vector $\bar{w}_{m}=\left(w_{1}, \ldots, w_{m}\right)^{T}$ includes the first $m$ components of $w$, and $\operatorname{diag}\left\{a_{i}\right\}_{m}=\operatorname{diag}\left\{a_{1}, \ldots, a_{m}\right\}$. After a substitution of $v(t)$ into (12) we arrive at

$$
\begin{gathered}
{\left[-C(T, x) \int_{0}^{T} \Phi(T, s) B(s) \operatorname{diag}\left\{\frac{u_{i}(s)}{x_{i}}\right\}_{m} d s+D(T, x)_{m},\right.} \\
\left.D(T, x)_{r-m}\right] w=E_{q_{0}, T}(u(\cdot), x) w=\eta .
\end{gathered}
$$

Hereabove subscripts $m, r-m$ refer, respectively, to the first $m$ and the last $r-m$ columns of $D(T, x)$. Finally, assuming invertibility of $E_{q_{0}, T}(u(\cdot), x)$, we compute the extended Jacobian inverse inverse

$$
\begin{aligned}
& \left(J_{q_{0}, T}^{E \#}(u(\cdot), x) \eta\right)(t)=\left(\begin{array}{c}
v(t) \\
w
\end{array}\right)= \\
& {\left[\begin{array}{cc}
-\operatorname{diag}\left\{\frac{u_{i}(t)}{x_{i}}\right\}_{m} 0 \\
I_{m} & 0 \\
0 & I_{r-m}
\end{array}\right] E_{q_{0}, T}^{-1}(u(\cdot), x) \eta .}
\end{aligned}
$$

It turns out that the inverse (13) has the following properties

$$
\begin{array}{r}
D H_{q_{0}, T}(u(\cdot), x) J_{q_{0}, T}^{E \#}(u(\cdot), x) \eta=0(\cdot), \quad \text { and } \\
J_{q_{0}, T}(u(\cdot), x) J_{i q_{0}, T}^{E \#}(u(\cdot), x) \eta=\eta
\end{array}
$$

of which the first guarantees repeatability, while the second provides a Jacobian right inverse.

Given a desirable point $y_{d} \in R^{r}$ in the taskspace, the inverse (13) defines an associated dynamic system

$$
\frac{d}{d \theta}\left(\begin{array}{c}
u_{\theta}(t) \\
x(\theta)
\end{array}\right)=-\gamma\left(J_{q_{0}, T}^{E \#}\left(u_{\theta}(\cdot), x(\theta)\right) e(\theta)\right)(t),
$$

where $\gamma>0$, and

$$
e(\theta)=K_{q_{0}, T}\left(u_{\theta}(\cdot), x(\theta)\right)-y_{d}
$$

stands for the taskspace error. We assume that the system (15) is complete, i.e. $u_{\theta}(\cdot)$ and $x(\theta)$ exist for every $\theta \in R$. Having differentiated the error along a trajectory of (15) and accomplished suitable substitutions we get

$$
\begin{aligned}
\frac{d}{d \theta} e(\theta)=J_{q_{0}, T}\left(u_{\theta}(\cdot), x(\theta)\right) \frac{d}{d \theta}\left(\begin{array}{c}
u_{\theta}(\cdot) \\
x(\theta)
\end{array}\right) & = \\
-\gamma J_{q_{0}, T}\left(u_{\theta}(\cdot), x(\theta)\right) J_{q_{0}, T}^{E \#}\left(u_{\theta}(\cdot), x(\theta)\right) e(\theta) & =-\gamma e(\theta) .
\end{aligned}
$$

The error vanishes exponentially, what means that the dynamic system (15) indeed defines an inverse kinematics algorithm. We shall call this algorithm hyperbolic. A solution of the inverse kinematic problem is obtained as the limit

$$
\left(\begin{array}{c}
u_{d}(t) \\
x_{d}
\end{array}\right)=\lim _{\theta \rightarrow+\infty}\left(\begin{array}{c}
u_{\theta}(t) \\
x(\theta)
\end{array}\right) .
$$


Observe that due to the first property of (14), when processing a sequence of inverse kinematic problems, all trajectories of (15) lie within an invariant manifold $V=\left\{(u(\cdot), x) \in X \mid H_{q_{0}, T}(u(\cdot), x)=H_{q_{0}, T}\left(u_{0}(\cdot), x_{0}\right)\right\}$, determined by the initial condition. These invariant manifolds coincide with integral manifolds of the distribution associated with the hyperbolic inverse kinematics algorithm.

\section{BAND-LIMITED EXTENDED JACOBIAN ALGORITHM}

For computational reasons we shall use a finitedimensional (Ritz) approximation of platform controls in the form of truncated trigonometric series,

$$
u_{i}(t)=\lambda_{0}^{i}+\sum_{k=1}^{s_{i}} \lambda_{2 k-1}^{i} \sin k \omega t+\lambda_{2 k}^{i} \cos k \omega t,
$$

for $i=1,2, \ldots, m, \omega=2 \pi / T$. This will be written concisely as $u(t)=P(t) \lambda$, where $\lambda=\left(\lambda^{1}, \lambda^{2}, \ldots, \lambda^{m}\right) \in$ $R^{s}, s=m+2 \sum_{i=1}^{m} s_{i}$, and $P(t)$ is a block diagonal matrix

$$
P(t)=\text { blocdiag }\left\{P_{s_{1}}(t), P_{s_{2}}(t), \ldots, P_{s_{m}}(t)\right\},
$$

whose $i$ th block entry

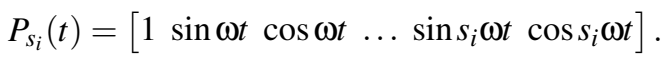

Because employing the truncated series automatically limits the bandwidth of control signals, we shall speak of band-limited controls. In the band-limited case the endogenous configuration gets finitely parameterized by $(\lambda, x) \in R^{s+p}$. The band-limited kinematics (2) will be denoted by $\tilde{K}_{q_{0}, T}(\lambda, x)$. The band-limited analytic Jacobian becomes an $r \times(s+p)$ matrix

$$
\tilde{J}_{q_{0}, T}(\lambda, x)=\left[C(T, x) \int_{0}^{T} \Phi(T, s) B(s) P(s) d s, D(T, x)\right] .
$$

The band-limited augmenting map (7) takes the form

$$
\tilde{H}_{q_{0}, T}(\lambda, x)=\left(x_{1} \lambda_{0}^{1}, \ldots, x_{1} \lambda_{s_{1}}^{1}, \ldots, x_{m} \lambda_{0}^{m}, \ldots, x_{m} \lambda_{s_{m}}^{m}\right) \text {. }
$$

Now, letting $u_{\theta}(t)=P(t) \lambda(\theta)$ and $x=x(\theta)$, we obtain the following band-limited extended Jacobian hyperbolic inverse kinematics algorithm

$$
\begin{gathered}
\frac{d}{d \theta}\left(\begin{array}{c}
\lambda(\theta) \\
x(\theta)
\end{array}\right)= \\
-\gamma\left[\begin{array}{cc}
-\operatorname{diag}\left\{\frac{\lambda^{i}(\theta)}{x_{i}(\theta)}\right\}_{m} & 0 \\
I_{m} & 0 \\
0 & I_{r-m}
\end{array}\right] \tilde{E}_{q_{0}, T}^{-1}(\lambda(\theta), x(\theta)) \tilde{e}(\theta),
\end{gathered}
$$

where $\tilde{e}(\theta)=\tilde{K}_{q_{0}, T}(\lambda(\theta), x(\theta))-y_{d}$ is the bandlimited taskspace error, and

$$
\begin{aligned}
\tilde{E}_{q_{0}, T}(\lambda, x)= & {\left[-C(T, x) \int_{0}^{T} \Phi(T, s) B(s) P(s) d s\right.} \\
& \left.\operatorname{diag}\left\{\frac{\lambda^{i}}{x_{i}}\right\}_{m}+D_{m}(T, x), D_{r-m}(T, x)\right] .
\end{aligned}
$$

It is easily seen that the first property of (14) results in the existence of hyperbolic invariant manifolds with respect to the dynamics of (16), defined as

$$
x_{i} \lambda^{i}=c_{i}=x_{i}(0) \lambda^{i}(0)
$$

for some constant vectors $c_{i} \in R^{2 s_{i}+1}, i=1,2, \ldots, m$. This justifies the adjective "hyperbolic" in our inverse kinematics algorithm. The invariant manifolds determine a relationship between the motion of the platform and of the onboard manipulator; thus by a proper choice of the invariant manifolds we may prescribe the motion coordination of a mobile manipulator during the accomplishment of its task. Furthermore, restricted to an invariant manifold, the hyperbolic inverse kinematics algorithm simplifies to the following form

$$
\begin{aligned}
& \frac{d x(\theta)}{d \theta}=-\gamma \tilde{E}_{q_{0}, T}^{-1}(\lambda(\theta), x(\theta)) \tilde{e}(\theta) \\
& \lambda^{i}(\theta)=\frac{c_{i}}{x_{i}(\theta)}, i=1, \ldots, m .
\end{aligned}
$$

The system (17) is well defined provided that $x_{i}(\theta) \neq$ 0 . It consists of a differential equation and an algebraic equation, what is computationally advantageous.

\section{SIMULATIONS}

In this section the band-limited hyperbolic inverse kinematics algorithm (16) will be applied to a mobile manipulator composed of a kinematic car-type mobile platform and an RTR aboard manipulator, portrayed in figure 1 . The coordinate vector $q=\left(q_{1}, q_{2}, q_{3}, q_{4}\right) \in$

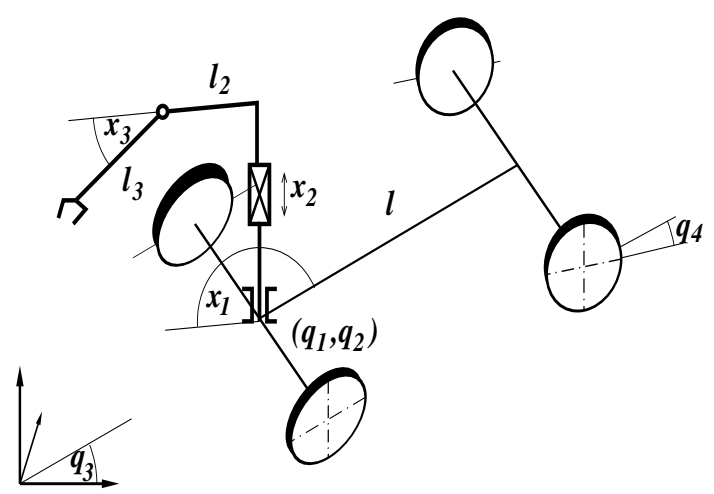

Fig. 1. RTR manipulator atop of kinematic car

$R^{4}$ is comprised of positions and orientations of the platform, and of the heading angle of the front wheels. The coordinate vector $x=\left(x_{1}, x_{2}, x_{3}\right) \in R^{3}$ includes manipulator joint positions. Cartesian positions of the end effector $y=\left(y_{1}, y_{2}, y_{3}\right) \in R^{3}$ serve as taskspace coordinates. The length of the car is taken as a measure unit $(l=1)$. The link lengths of the onboard manipulator are equal to $l_{2}=0.5, l_{3}=1$. The resulting control system representation (1) of kinematics, excluding lateral slip of platform wheels, takes the following form 


$$
\begin{gathered}
\left\{\begin{array}{c}
\dot{q}_{1}=u_{1} \cos q_{3} \cos q_{4}, \\
\dot{q}_{2}=u_{1} \sin q_{3} \cos q_{4}, \\
\dot{q}_{3}=u_{1} \sin q_{4}, \\
\dot{q}_{4}=u_{2},
\end{array}\right. \\
y=\left(\begin{array}{l}
y_{1} \\
y_{2} \\
y_{3}
\end{array}\right)=\left(\begin{array}{c}
q_{1}+\left(l_{2}+l_{3} \cos x_{3}\right) \cos \left(q_{3}+x_{1}\right) \\
q_{2}+\left(l_{2}+l_{3} \cos x_{3}\right) \sin \left(q_{3}+x_{1}\right) \\
x_{2}+l_{3} \sin x_{3}
\end{array}\right) .
\end{gathered}
$$

In simulations we set $T=1$, and use very simple platform controls, of the form

$$
u_{i}(t)=\lambda_{0}^{i}+\lambda_{1}^{i} \sin 2 \pi t+\lambda_{2}^{i} \cos 2 \pi t, \quad i=1,2 .
$$

It may be easily checked that, after replacing the continuous version (16) of the hyperbolic algorithm by a discrete one, the algorithm's dynamics may no longer stay within the invariant manifold. For this reason, in order to define the discrete form of the hyperbolic algorithm we need to use instead of (17) the general formula (16). The result is the following

$$
\begin{gathered}
x_{\theta+1}=x_{\theta}-\gamma \tilde{E}_{q_{0}, T}^{-1}\left(\lambda_{\theta}, x_{\theta}\right) \tilde{e}_{\theta}, \\
\lambda_{\theta+1}^{1}=\lambda_{\theta}^{1}\left(2-\frac{x_{1 \theta+1}}{x_{1 \theta}}\right), \\
\lambda_{\theta+1}^{2}=\lambda_{\theta}^{2}\left(2-\frac{x_{2 \theta+1}}{x_{2 \theta}}\right) .
\end{gathered}
$$

For illustration of the algorithm's performance we have solved an exemplary inverse kinematics problem of reaching the taskspace point $y_{d}=(0,0,1)$ from two initial platform postures $q_{0}=(-9,0,0,0)$, $q_{0}=(-9,3,0,0)$, with fixed initial configuration $x_{0}=$ $(\pi / 2,0.5, \pi / 2)$ of the onboard manipulator. The platform control functions contain either constant terms $\left(s_{1}=s_{2}=0\right)$ or constant terms plus first order harmonics $\left(s_{1}=s_{2}=1\right)$. In the former case $\lambda_{0}=$ $(1,-0.1)$, in the latter the initial amplitudes of harmonics are set to $\lambda_{0}=(1,1,1,-0.1,-0.1,-0.1)$. Figures 2,3 show results of computer simulations.

\section{CONCLUSION}

Using the endogenous configuration space approach we have derived a hyperbolic extended Jacobian, repeatable inverse kinematics algorithm for mobile manipulators. By means of a finite-dimensional approximation of platform controls a band-limited version of this algorithm has been obtained. The continuous band-limited algorithm operates on invariant manifolds in the configuration space, whose existence makes the algorithm repeatable. Computer simulations accomplished for a discrete formulation of the hyperbolic algorithm show that its convergence and performance remain satisfactory. However, as we have already noticed, the discrete hyperbolic algorithm's dynamics abandon the invariant manifold, rendering the algorithm unrepeatable. In order to restore repeatability, the result of each iteration of the algorithm should be projected back onto the invariant manifold determined by the initial condition. It turns out that in order to endow the hyperbolic algorithm with the projection it suffices to solve a 4 th order polynomial
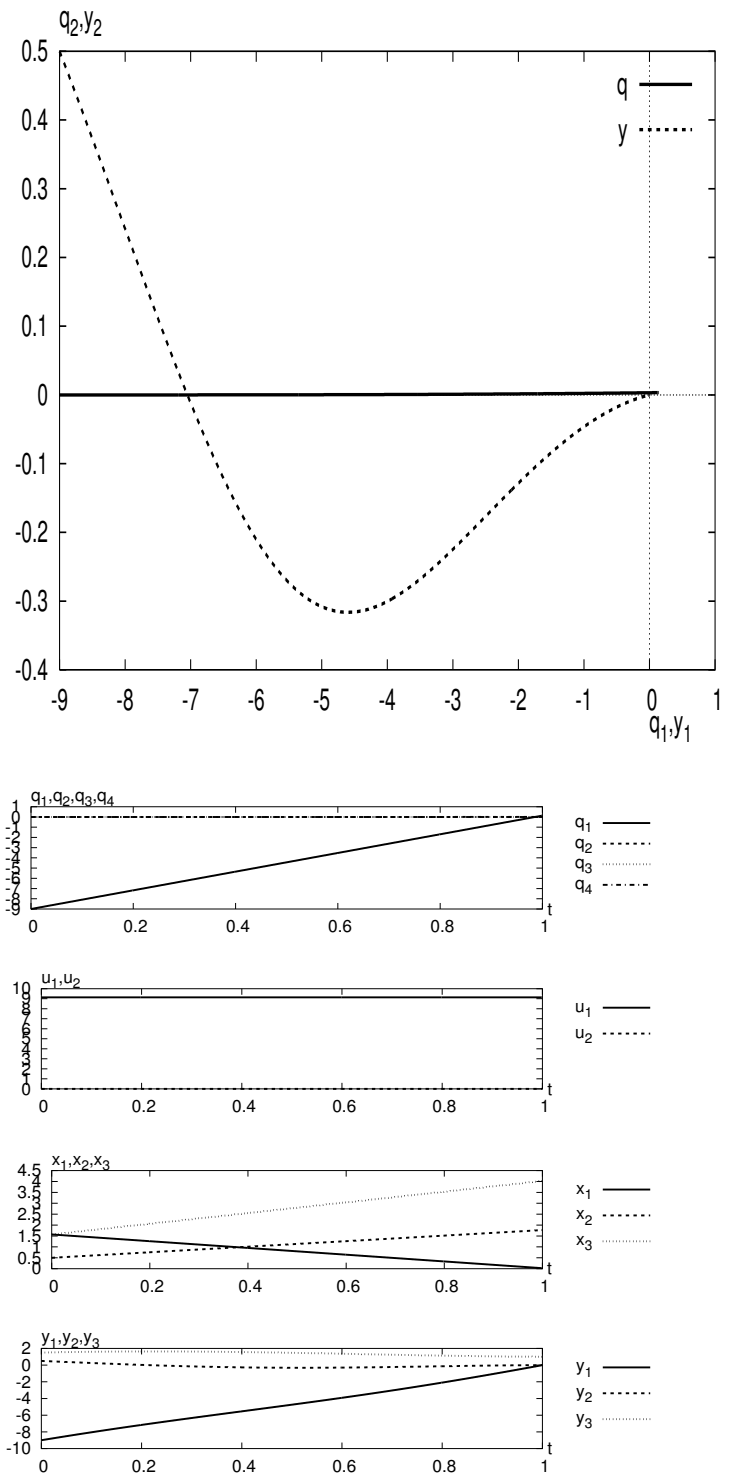

Fig. 2. Solution to the inverse kinematics problem. Initial data: $q_{0}=(-9,0,0,0), x_{0}=(\pi / 2,0.5, \pi / 2)$, $\lambda_{0}=(1,-0.1)$. Convergence: number of iterations 50, final taskspace error 9.18774e -11 .

equation in joint positions of the onboard manipulator, which can be done analytically. On the other hand, convergence of the algorithm with projection needs further investigations.

It should be observed that the hyperbolic inverse kinematics algorithm is well defined provided that positions of certain joints of the aboard manipulator stay away from 0 . As a matter of fact, this disadvantage can be removed easily by replacing $x_{i}$ in (7) by a term like $x_{i}^{2}+\varepsilon$ for a positive number $\varepsilon$. Obviously, the new algorithm will no longer be hyperbolic.

A feature of extended Jacobian inverse kinematics algorithms is the existence of invariant manifolds in the endogenous configuration space of a mobile manipulator. We believe that these invariant manifolds will play a significant role in providing a proper motion coordination between the platform and the onboard 

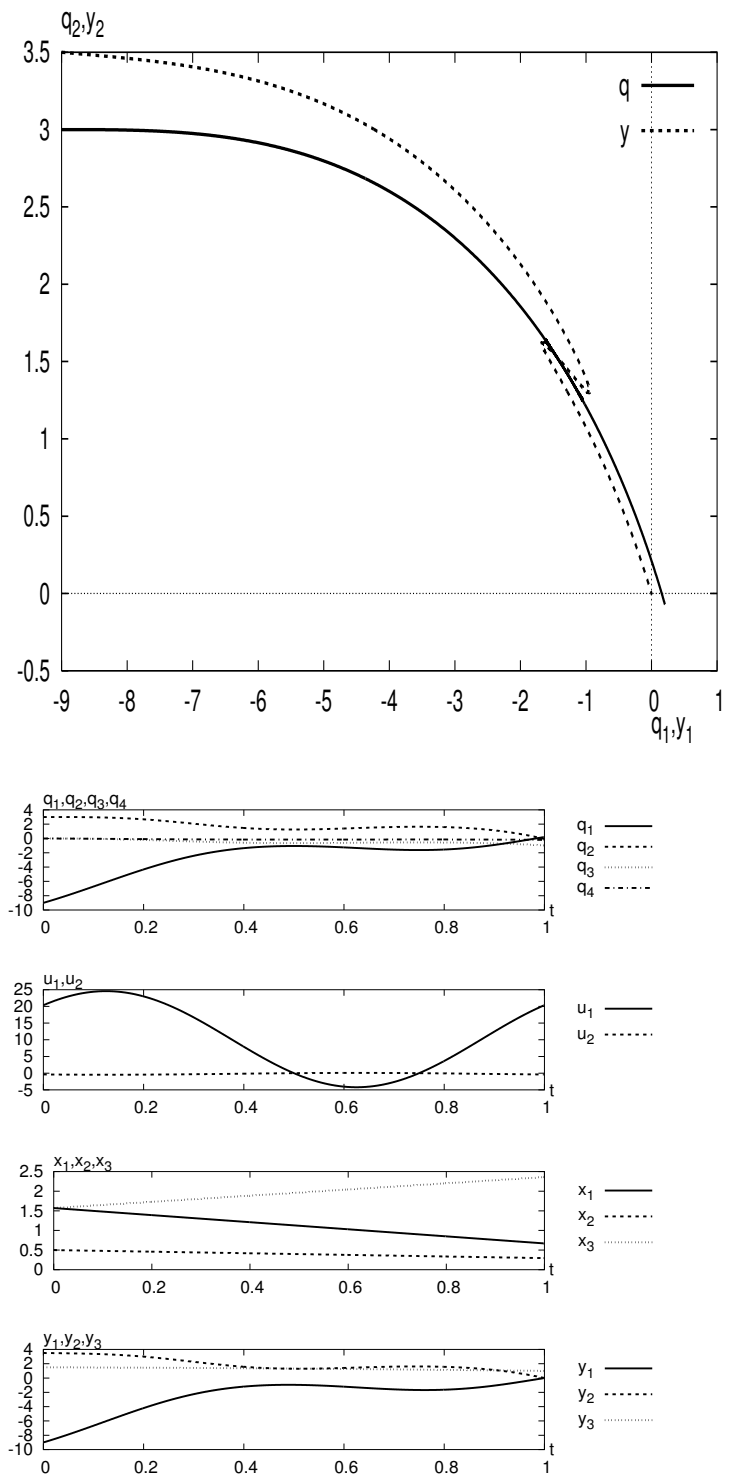

Fig. 3. Solution to the inverse kinematics problem. Initial data: $q_{0}=(-9,3,0,0), x_{0}=(\pi / 2,0.5, \pi / 2)$, $\lambda_{0}=(1,1,1,-0.1,-0.1,-0.1)$. Convergence: number of iterations 500, final taskspace error $1.46634 e-10$.

manipulator. This question as well as the question of restoring repeatability by endowing the algorithm with a projection will be a subject of our future research.

\section{ACKNOWLEDGMENTS}

This research has been supported by the Polish State Committee of Scientific Research.

\section{REFERENCES}

Baillieul, J. (1985). Kinematic programming alternatives for redundant manipulators. In: Proc. 1985 IEEE Int. Conf. Robot. Automat. St. Louis, LA. pp. 818-823.
Bayle, B., J.-Y. Fouquet and M. Renaud (2003). Manipulability of wheeled mobile manipulators: Application to motion generation. Int. J. Robotics Res. 22, 564-581.

Duleba, I. and J. Z. Sasiadek (2003). Nonholonomic motion planning based on Newton algorithm with energy optimization. IEEE Trans. Contr. Syst. Technology 11, 355-363.

Galicki, M. (2003). Inverse kinematics solution to mobile manipulators. Int. J. Robotics Res. 22, 10411064.

Mazur, A. (2004). Hybrid adaptive control laws solving a path following problem for nonholonomic mobile manipulators. Int. J. Control 77, 12971306.

Papadopoulos, E. and S. Dubowsky (1991). Coordinated manipulator/spacecraft motion for space robotics. In: Proc.1991 IEEE int. Conf. Robot. Automat. Sacramento, CA. pp. 1696-1701.

Roberts, R. G. and A. A. Maciejewski (1992). Nearest optimal repeatable control strategies for kinematically redundant manipulators. IEEE Trans. Robot. Automat. 8, 327-337.

Tchoń, K. (2002). Repeatability of inverse kinematics algorithms for mobile manipulators. IEEE Trans. Aut. Contr. 47, 1376-1380.

Tchoń, K. and J. Jakubiak (2003). Endogenous configuration space approach to mobile manipulators: a derivation and performance asse ssment of Jacobian inverse kinematics algorithms. Int. J. Control 76, 1387-1419.

Tchoń, K. and R. Muszyński (2000). Instantaneous kinematics and dexterity of mobile manipulators. In: Proc. 2000 IEEE Int. Conf. Robot. Automat. San Francisco, CA. pp. 2493-2498.

Tchoń, K., J. Jakubiak and R. Muszyński (2003). Regular Jacobian motion planning algorithms for mobile manipulators. In: Proc. 15th IFAC World Congress. Vol. K. Barcelona, Spain. pp. 231-236. 